\title{
Les participants et les organisateurs du Forum social mondial : la diversité du militantisme
}

Odaci Luiz Coradini

\section{(2) OpenEdition \\ 1 Journals}

Édition électronique

URL : http://journals.openedition.org/conflits/12673

DOI : $10.4000 /$ conflits. 12673

ISSN : $1777-5345$

Éditeur :

CCLS - Centre d'études sur les conflits lilberté et sécurité, L'Harmattan

Édition imprimée

Date de publication : 20 juillet 2008

Pagination : 153-175

ISBN : 978-2-296-05909-2

ISSN : 1157-996X

Référence électronique

Odaci Luiz Coradini, «Les participants et les organisateurs du Forum social mondial : la diversité du militantisme », Cultures \& Conflits [En ligne], 70 | été 2008, mis en ligne le 04 janvier 2010, consulté le 30 mars 2021. URL : http://journals.openedition.org/conflits/12673 ; DOI : https://doi.org/10.4000/ conflits. 12673 


\section{Les participants et les organisateurs du Forum social mondial : la diversité du militantisme}

\section{Odaci LUIZ CORADINI}

Odaci Luiz Coradini est professeur de sciences sociales et chercheur à l'Universidade Federal de Rio Grande do Sul, au Brésil. Ses recherches portent notamment sur des sujets relatifs au militantisme, à la participation et à la représentation politiques.

\footnotetext{
Ce texte s'attache à présenter les premiers résultats d'une étude toujours en cours menée sur le 5e Forum social mondial (FSM), qui s'est déroulé à Porto Alegre du 26 au 31 janvier 2005. Plusieurs axes d'analyse ont présidé à cette étude. Nous nous sommes d'abord intéressés aux significations que peuvent avoir des réferentiels idéologiques, tels que le tiers-mondisme ou l'altermondialisme, dans des conditions diverses. Puis nous avons étudié les modalités des relations qu'entretiennent les participants et les organisateurs du FSM avec l'espace politique.
}

Le matériel empirique sur lequel s'appuie cette étude se compose de 36 interviews de représentants d'organisations présentes au FSM, d'une documentation fournie sur ces mêmes organisations et les parcours sociaux de leurs directeurs respectifs, et enfin d'un total de 2540 questionnaires auxquels des participants ont bien voulu répondre ${ }^{1}$.

1. Les organisateurs du $5^{\mathrm{e}} \mathrm{FSM}$ ont permis la mise en avant de quelques questions importantes, notamment celles des origines sociales et des modalités d'engagement et de militantisme. Cet ensemble de questionnaires repose sur un échantillon considérant les différentes catégories de participants, il est statistiquement représentatif des porte-paroles d'ONG au Brésil, avec la plus grande diversité possible d'aspects et de thématiques représentés. Les entretiens avaient pour axes principaux, outre les origines et le parcours professionnel, le parcours militant et la position dans les oppositions existant au FSM et face à l'espace politique. Enfin, nous avons utilisé un matériel en version imprimée (format papier) et en version électronique. Il s'agit généralement de textes qui, en plus de viser à la diffusion d'idées et de renseignements sur les organisations, ont pour but de faire l'éloge de leaders ou de militants. Il s'agit donc les utiliser avec prudence, en tenant compte des conditions, intérêts et logiques d'action sous-tendant leur production et leur diffusion. Par ailleurs, il s'agit de productions de militants témoignant d'un 
En vue d'une meilleure qualification du matériel utilisé, il convient de souligner, en premier lieu, que l'importance et la complexité de ce type d'événements imposent des limites à la recherche, sans compter le risque de glisser vers une apologie du militantisme liée au travail de rassemblement d'informations. Contrairement à ce que l'on pourrait croire a priori, le fait que le FSM soit un vaste rassemblement de militants rend le travail ardu, particulièrement parce que ces derniers maintiennent une certaine distance, voire une certaine méfiance, à l'égard des chercheurs et de leur travail. Ils considèrent en effet que les critères nécessaires à l'appréhension et l'évaluation des acteurs et du sujet étudié dans le cadre de ce type d'études relèvent d'une démarche politique guère appréciée dans ce genre de millieu ${ }^{2}$. Le nombre d'acteurs, la diversité thématique ainsi que la situation générale de foisonnement et d'ébullition due aux multiples activités des participants et des organisateurs, sont autant de contraintes à prendre en compte afin de s'assurer un bon contrôle des informations, obtenues au moyen d'observations et d'interviews. Si une grande quantité d'informations circule au FSM (l'échange et la diffusion d'informations constituant d'ailleurs l'un de ses objectifs principaux), celles-ci ne concernent que les aspects suscitant l'intérêt et pour la diffusion desquels des moyens conséquents sont mobilisés. Les informations sur les modalités et conditions d'engagement et de militantisme obéissent bien évidemment à d'autres intérêts, leur obtention dépendant de la forte concurrence entre organisations et acteurs, ce qui rend celle-ci assez inégale. Soulignons enfin que les conditions de participation et règles de fonctionnement des FSM diffèrent d'une année à l'autre. Il convient donc de resituer plus précisément ce $5^{\mathrm{e}} \mathrm{FSM}$ dans son contexte.

\section{Le FSM comme rassemblement de militants. Modalités de partici- pation}

Elaboré à partir des suggestions formulées par les organisations participantes, le programme de ce FSM reposait sur 11 espaces thématiques qui structuraient les différentes activités ${ }^{3}$. Lors de la marche qui a marqué le

effort pour se démarquer les uns des autres. Tout cela finit par faire du FSM un milieu propice au recueil d'un certain nombre d'informations qui, complétées par d'autres sources, peuvent s'avérer utiles.

2. Sur les relations entre la production et le contrôle d'informations biographiques et la formation du capital politique, voir Pennetier C., Pudal B. (dirs.), Autobiographies, antocritiques, aveux dans le monde communiste, Paris, Belin, 2002, particulièrement pp. 21-27; et sur les difficultés spécifiques de l'étude d'ONG, voir Siméant J., Dauvin P. (dirs.), ONG et Humanitaire, Paris, L'Harmattan, 2004.

3 . Les « espaces» sont les suivants: Affirmation et défense des biens communs de la terre et des peuples - Alternative à la mercantilisation et au contrôle des transnationales; Art et création : construire les cultures de résistance des peuples; Communication : pratiques contre-hégémoniques, droits et alternatives; Défendre les diversités, la pluralité et les identités ; Droits de l'Homme et dignité pour un monde juste et égalitaire; Economies souveraines par et pour les peuples - Contre le capitalisme néo-libéral; Ethique, cosmovisions et spiritualités Résistances et défis pour un nouveau monde ; Luttes sociales et alternatives démocratiques Contre la domination néo-libérale ; Paix et démilitarisation - Lutte contre la guerre, le libre- 
début du Forum, on a compté plus de 200000 personnes présentes. Au total, il a eu près de 155000 participants inscrits. Environ 6872 organisations venant de 151 pays différents ont été engagées dans plus de 2500 activités, distribuées entre les 11 espaces thématiques du « Territoire social mondial ». Les délégations les plus importantes venaient du Brésil, de l'Argentine, des EtatsUnis, de l'Uruguay et de la France. Près de 500000 personnes au total ont ainsi circulé sur ce «Territoire social mondial ${ }^{4}$.

Le lieu où se déroule le FSM est un élément déterminant pour la participation des militants. Le facteur géographique a en effet, comme nous le verrons, des effets à la fois sur le nombre de participants et sur la composition même du public. A l'instar des trois premiers FSM, le $5^{\mathrm{e}}$ forum s'est tenu à Porto Alegre, le 4e FSM ayant eu lieu à Mumbai, en Inde, en 2004. On a pu constater une croissance constante du nombre de participants depuis 2001 :

\begin{tabular}{|c|c|}
\hline Année du FSM & Nombre de participants \\
\hline 2001 & 50000 \\
\hline 2002 & environ 20 000 \\
\hline 2003 & $\begin{array}{c}100000 \\
\text { (plus environ 25 000 par- } \\
\text { ticipants au Campement } \\
\text { de la jeunesse) }\end{array}$ \\
\hline 2004 & 74126 \\
\hline 2005 & 155000 \\
\hline
\end{tabular}

Outre les effets de la relative proximité géographique, la composition des FSM dépend directement de leurs conditions d'accès. Ces dernières ont connu des modifications importantes à l'occasion du $5^{\mathrm{e}} \mathrm{FSM}$, avec notamment l'introduction d'un "processus de consultation et d'agglutination 5 » ainsi qu'une plus grande importance accordée aux activités « autogérées ». Le programme,

échange et la dette ; Pensée indépendante, réappropriation et socialisation de la connaissance (des savoirs) et des technologies ; Vers la construction d'un ordre démocratique international et intégration des peuples. Les « axes transversaux » sont les suivants : Emancipation sociale et dimension politique des luttes; Lutte contre le capitalisme patriarcal ; Luttes contre le racisme et autres formes d'exclusion basées sur l'ascendance ; Genre ; Diversité. Pour plus de détails sur les espaces et activités, on peut consulter le site :

http://www.forumsocialmundial.org.br/, page consultée le 20 octobre 2005.

4. Ibid.

5. Ibid. 
élaboré à partir des suggestions présentées par les organisations participantes, s'est donc composé de 11 «espaces » thématiques, de 3 «espaces transversaux » et d'un ensemble d' «activités». L'une des principales nouveautés introduite dans ce FSM a très certainement été la possibilité de participer au forum sans être lié à une quelconque organisation, ce qui, bien évidemment, n'est pas resté sans effets sur la composition du public.

\section{Bases institutionnelles et principes de légitimation des organisa- tions promouvant le FSM}

Exceptée la présence de quelques organisations internationales bien connues, les trois premiers FSM ont été à la charge d'un comité organisateur composé de 8 organisations brésiliennes. Pour le $5^{\mathrm{e}} \mathrm{FSM}$, celui-ci était constitué de 23 organisations, réparties en 8 groupes de travail, toutes chargées de sa promotion ${ }^{6}$. Ce comité, tout comme ses prédécesseur, fonde son travail sur la Charte de principes du Forum social mondial, élaborée lors du 1er FSM, en 2001 à Porto Alegre ${ }^{7}$.

Lorsqu'on tente de visualiser cet immense ensemble d'organisations, de publics, de sujets et d'oppositions, la première tentation est d'y chercher un dénominateur commun qui puisse servir à formuler une première définition du FSM, même très générale. Dans le cas des autres forums de ce type, mieux étudiés, (comme c'est le cas particulièrement du $2^{\mathrm{e}}$ Forum social européen, en 2003 à Paris), l'« altermondialisme » a été pris comme le principal élément de définition, bien qu'il se maintienne au niveau général d'une " nébuleuse », caractérisée par la «diversité d'organisations et thématiques 8 . Dans ce cas précis, ni la grande quantité de témoignages d'acteurs portant sur la définition

6. Les organisations qui composent ce comité organisateur brésilien sont les suivantes : ABONG Association brésilienne d'organisations non gouvernementales; AMB - Association de femmes brésiliennes ; ATTAC - Association pour la taxation des transactions financières pour l'aide aux citoyennes et citoyens; CONAM - Confédération nationale des associations d'habitants ; Caritas Brésil ; CAT - Centrale indépendante des travailleurs ; CBJP - Commission brésilienne de justice et paix de CNBB (Conférence nationale des évêques du Brésil); Cives - Association brésilienne d'entrepreneurs par la citoyenneté; Clacso - Consejo Latinamericano de Ciencias Sociales; CMP - Centrale de mouvements populaires ; Comité organisateur du campement intercontinental de la jeunesse; Comité afro du FSM ; CUT - Centrale unique des travailleurs ; FBOM - Forum brésilien des ONG et mouvements sociaux pour l'environnement et le développement ; GTA Groupe de travail amazonien ; Ibase - Institut brésilien d'analyses sociales et économiques ; IPF Institut Paulo Freire ; Jubilé Sud Brésil ; Marche mondiale des femmes ; MST - Mouvement des travailleurs agricoles sans terre ; Réseau social de justice et droits humains ; UJS - Union de la jeunesse socialiste ; UNE - Union nationale des étudiants.

7. Voir "Carta de princípios do Fórum Social Mundial”, sur le site : http://www.forumsocialmundial.org.br, page consultée le 11 octobre 2007.

8. Voir Gobille B., Uysal A., " Cosmopolites et enracinés », in Agrikoliansky E., Sommier I. (dirs.), Radiographie du mouvement altermondialiste: le second Forum social européen, Paris, La Dispute, 2005, p. 106 ; Agrikoliansky E, Sommier I., Hajji I., « Formes et publics de l'altermondialisme ", in Agrikoliansky, E ; Sommier, I. (dirs.), op. cit., p. 293. Pour des études sur différents aspects du $2^{\mathrm{e}}$ FSE, voir Agrikolianky E., Sommier I., op. cit. Sur l'altermondialisme en France, voir Agrikoliansky E., Fillieule, O., Mayer N. (dirs.), L'Altermondialisme en France: la longue histoire d'une nouvelle cause, Paris, Flammarion, 2005 ; Sommier I., Le 
et l'organisation des FSM, ni la grande hétérogénéité des thèmes et organisations participantes n'autorisent de définition a priori du FSM. Les auteurs de l'étude collective menée sur la participation de mouvement «altermondialiste » français au 2e FSE soulignent la nécessité de poursuivre les recherches centrées sur le contexte national, afin d'éviter la généralisation d'aspects internationaux pris hors contexte 9 . Cependant, lorsqu'on considère une certaine configuration nationale, les axes d'opposition qui organisent les significations et les critères de définition se modifient. Ainsi, les catégories basiques qui s'opposent dans cet ensemble de mobilisations s'insèrent dans d'autres systèmes d'action, ce qui signifie que des "ensembles de regroupements et d'associations contribuent, chacun à leur façon et selon leurs propres logiques de financement, à la construction des groupes de référence $10 »$. Tel est le cas, par exemple, des sens spécifiques que revêtent, pour la plupart des organisateurs et des participants brésiliens, les références au catholicisme, aux différents types de philanthropie (d'entreprise ou des églises notamment), à la politique et aux autres valeurs et pratiques sociales, qui diffèrent clairement des significations qui peuvent leur être acccordées ailleurs, notamment dans les sociétés des pays développés. Ces différentes perspectives et bases sociales n'empêchent cependant pas l'existence d'une codification générale sur le plan des prises de position plus officielles, d'une règle venant surplomber les oppositions, lesquelles participent à la dynamique même du FSM.

Parmi les définitions possibles de l'objet étudié, le FSM n'est saisi qu'en tant que processus de constitution et catalyseur de modalités d'engagement et de militantisme. Cela implique que l'on tienne d'abord compte des relations, non seulement entre les diverses modalités d'engagement, mais aussi entre la diversité des sphères et conditions sociales en jeu (avec des organisations reposant sur des limites et des principes de légitimation syndicales ou "de classe », humanitaires, politiques, religieux, entre autres) et leurs affinités et facteurs d'exclusion. Il faut ensuite se pencher sur les liens existant entre ces modalités d'engagement et de militantisme et les conceptions de l'espace politique. Cela implique enfin de considérer les relations centre/périphérie et leurs effets sur les modalités d'engagement et de militantisme ${ }^{11}$. L'hypothèse générale repose sur l'idée que

Renouveau des mouvements contestataires à l'beure de la mondialisation, Paris, Flammarion, 2003 et le numéro spécial de la Revue française de science politique, vol.54, $\mathrm{n}^{\circ} 3$, juin 2004, consacré aux relations entre les ONG et l'altermondialisme.

9. Agrikoliansky E., Sommier I., Hajji I., "Formes et publics de l'altermondialisme », in Agrikoliansky E., Sommier I., Hajji I. (dirs.), op. cit., pp. 10-11.

10. Lagroye J., François B., Sawicki F., Sociologie politique, $4^{\mathrm{e}}$ éd., Paris, Presses de Sciences Po / Dalloz, 2002, p. 271.

11. Il ne s'agit donc pas d'un travail consacré spécifiquement aux « mouvement sociaux », bien qu'il n'exclue pas les instruments résultant des avancées obtenues dans ce type d'études. Cela n'exclut pas cependant les différences relatives aux présupposés et à l'usage de tels instruments, qui, plus qu'une Charte de principes, comprend des différences concernant les agendas de recherche. Bien que cela ne soit pas l'objet du présent texte, il convient d'indiquer rapidement que, malgré les avancées théoriques et méthodologiques des études sur les « mouvements sociaux » 
des rassemblements tels que le FSM dépendent d'une grande ouverture dans leurs principes et critères d'insertion, ouverture qui est la condition même de leur efficacité. Il reste que l'hétérogénéité créée par cette ouverture est également la source des ambivalences et perpétuelles redéfinitions du FSM.

\section{Altermondialisme, tiers-mondisme et autres principes de regroupe- ment et de mobilisation}

Comme cela a été mentionné lors de l'analyse d'événements semblables (comme le $2^{\mathrm{e}} \mathrm{FSE}$ ), le mouvement altermondialiste, malgré ses définitions plutôt floues et indéterminées, est généralement pris comme l'une des principales bases idéologiques de ces événements. L'altermondialisme français a d'ailleurs participé à la création d'une nouvelle configuration dans les relations entre l'engagement associatif, syndical, ainsi qu'à la recomposition de référentiels qui, jusqu'alors, avaient tendance à s'opposer. Ce serait le cas, par exemple, de certains pôles de la mouvance catholique, qui se seraient alliés avec des mouvements de gauche donnant lieu à une " association entre militants communistes, trotskystes [...] et la fraction la plus progressiste de l'Eglise catholique », et par le renforcement d'organisations altermondialistes comme ATTAC ${ }^{12}$. Cependant, il ne s'agit là que de l'examen du cas français. Bien que l'affirmation récente de l'altermondialisme à une échelle transnationale soit une réalité, la comparaison avec d'autres configurations nationales doit tenir compte des particularités de l'opposition historique entre les défenseurs du républicanisme, ou de la « gauche », et les catholiques. Par ailleurs, il faut considérer les effets différenciés de la nouvelle configuration liée à la fin de la Guerre froide pour comprendre les pri-

dans la littérature nord-américaine, ceux-ci y sont généralement saisis en tant qu'objet ou champ d'étude propres (pour des exemples récents, voir notamment Giugni M., McAdam D., Tilly C. (eds.), How Social Movements Matter, Minneapolis, University of Minnesota Press, 1999). Plus récemment, l'une des principales avancées méthodologiques a été l'adoption d'approches plus relationnelles ou processuelles, ou reposant sur des études de réseaux (notamment Diani M., McAdam D. (eds.), Social Movements and Networs. Relational Approches to Collective Action, Oxford, Oxford University Press, 2003). L'une des conséquences de cette tendance est l'importance croissante accordée aux conditions individuelles d'engagement ou de désengagement qui, dans les conditions actuelles de la connaissance de situations comme celles d'Amérique latine, ne semble pas mériter une place centrale dans la recherche. Par ailleurs, sur une autre ligne de recherche, ont été publiés récemment plusieurs travaux portant explicitement sur le problème des relations de différentes modalités d'engagement avec l'espace politique (voir entre autres Goldstone J.A. (ed.), States, Parties, and Social Movements, Cambridge, Cambridge University Press, 2003 ; Jenkins J.C., Klandermans B. (eds.), The Politics of Social Protest. Comparative Perspectives on State and Social Movements, London, University College London, 1995). L'un des principaux problèmes de cette bibliographie tient à la proximité de la plupart des travaux avec les présupposés du «modèle pluraliste ", c'est-à-dire une série de prémices d'un point de vue propre aux sciences sociales, mais également selon une situation historique comprenant certaines configurations nationales. Le problème principal est que le "modèle pluraliste », en tant que réalité historique, ne s'accorde pas aux situations latino-américaines, "périphériques ", relevant d'autres modes de recrutement d'élites et de relations avec l'univers de la politique (voir notamment Badie B., " Le modèle centre-péripherie ", Le Développement politique, $5^{\mathrm{e}}$ éd., Paris, Economica, 1994).

12. Agrikoliansky E., Fillieule O., Mayer N. (dirs.), op. cit., pp. 46-56. 
ses de position relatives aux relations centre/périphérie et le retour de l'utilisation de la philanthropie, dans ses différentes modalités, comme moyen de domination et de légitimation ${ }^{13}$. L'une de ces conséquences les plus directes est le fait que les relations entre l'engagement dans les ONG et d'autres formes d'engagement et de militantisme, dans les pays comme le Brésil, deviennent plus ambivalentes et diversifiées ${ }^{14}$.

Aussi, selon les observateurs du $2^{\mathrm{e}} \mathrm{FSE}{ }^{15}$, il est surprenant de constater par exemple que 2 des 8 organisations du comité organisateur du FSM de 2004 seraient rattachées à l'Eglise catholique, ou encore que la position de Caritas Brésil serait à l'opposé de celle de Caritas Europe. En effet, la participation de la première au FSM résulterait de la position centrale de l'Eglise catholique dans les «mouvements sociaux»16. Il s'agirait d'un contraste lié à une configuration nationale, comme celle de la France, où historiquement des catégories comme «politisation», «gauche» et, par extension, « engagement» et «militantisme », sont directement associées à certaines positions politiques et idéologiques placées dans le pôle opposé aux croyances religieuses. Dans le cas du FSM, non seulement la quantité d'organisations rattachées au catholicisme est plus importante mais, en outre, une bonne partie de leurs dirigeants présente un parcours social fortement associé à des institutions et à un engagement religieux, dans une conjoncture largement favorable à l'intervention des églises dans l'univers associatif et politique. Outre la sphère scolaire ${ }^{17}$, la présence de l'Eglise peut se manifester via certaines institutions, ou de manière plus indirecte, sous forme de philosophie sociales et politiques. Autant de façons différentes de s'approprier le catholicisme et d'établir un lien entre religion et politique, comme l'indique J.M. Donegani 18.

Ainsi, le seul nombre d'institutions ne saurait constituer un bon indicateur des influences religieuses ou d'autres composantes. D’une manière générale, les oppositions entre organisations et principes d'adhésion à des modalités d'engagement sont très nombreuses. Ces oppositions sont observables dans la Charte

13. Sur les relations centre/périphérie, voir Dezalay Y., Garth B.G., La Mondialisation des guerres de palais: la restructuration du pouvoir d'Etat en Amérique latine, entre notables du droit et "Chicago Boys", Paris, Seuil, 2002 ; Badie B., Le Développement politique, Paris, Economica, 1994.

14. Voir Arantes P.E., «Equerda e direita no espelho das ONGs », Cadernos Abong, São Paulo, $\mathrm{n}^{\circ} 27$, mai 2000.

15. Agrikoliansky E., Sommier, I. (dirs.), op. cit.

16. du Cleziou Y.R., Johsua F., «Entre radicalité et apoliticisme », in Agrikoliansky E., Sommier I. (dirs.), op. cit., p. 246.

17. Sur l'enseignement théologique et ses relations avec le renforcement du tiers-mondisme et les utilisations des sciences sociales, voir Luiz Coradini O., "L'enseignement universitaire de théologie, les rapports centre/périphérie et les usages des sciences humaines et sociales », Cabiers de la recherche sur l'éducation et les savoirs, 2008 (à paraître).

18. Donegani J.M., La Liberté de choisir : pluralisme religieux et pluralisme politique dans le catholicisme français contemporain, Paris, Presses de la Fondation nationale des sciences politiques, 1993, pp. 16-23. 
de principes évoquée plus haut et dans les luttes internes pour le contrôle et l'imposition de critères de classement, plus particulièrement dans la définition même du FSM, comme « espace » en opposition à « mouvement ». Cela transparaît d'ailleurs dans les témoignages des acteurs clefs ${ }^{19}$. Les oppositions internes au FSM montrent que sa définition en tant qu' « espace » est, d'une part, associée aux nouvelles technologies de mobilisation, particulièrement d'origine nord-américaine (comme celle des « réseaux»), mais aussi à des conceptions catholiques, plutôt qu’à des principes plus politiques, liés à la défense de l'idée de «mouvement ». Dans la mesure où la base institutionnelle se rattache à une église ou à une ONG, et étant donné que le FSM est constitué essentiellement de «mobilisateurs de mobilisateurs ", il peut être plus pratique d'être promoteur d'un « espace » au nom de principes généraux que d'un «mouvement » politiquement délimité, et donc restreint.

Dans ces conditions, bien qu'il n'y ait pas d'orientation politique ou de «mouvement» qui unifie les promoteurs et le public, à l'exception d'une opposition généralisée à la « mondialisation » (dont la signification varie, là aussi, selon les circonstances), quelques principes d'inclusions et de divisions internes transparaissent. Parmi les organisations brésiliennes - que l'on considère les 8 organisations initialement présentes en 2001 ou de celles, plus nombreuses, présentes en 2005 (au nombre de 23) - quelques-uns des pôles se dessinent en fonction des modalités d'organisation, des types d'engagement (associatif, syndical, étudiant) et des principes de légitimation. Comme nous le verrons par la suite, cette polarisation organise les oppositions et les divisions au sein des participants, malgré, bien évidemment, des poids divers dans l’organisation et dans les prises de décisions.

Le premier pôle relève de la sphère du syndicalisme, dont les principaux représentants sont la CUT (Centrale unique des travailleurs), les fédérations de syndicats associées et la CAT (Centrale indépendante des travailleurs). Il s'agit donc d'un ensemble plus directement associé à la « gauche » dans le champ syndical (et au PT - Parti des travailleurs dans l'espace politique) et d'une autre, la CAT, proche d'une orientation «chrétienne ». Le deuxième pôle principal est formé par les ONG nationales ou locales, parmi lesquelles se détachent l’ABONG

19. Pour obtenir des données empiriques plus détaillées, voir les témoignages de Bernard Cassen : Cassen B., Tout a commencé à Porto Alegre... Mille forums sociaux !, Paris, Mille et une nuits, 2003, et de Whitaker C.O., Desafio do Fórum Social Mundial [Le défi du Forum social mondial], São Paulo, Ed. Fundação Perseu Abramo/Edições Loyola, 2005, www.forumsocialmundial.org.br. Sur les conceptions divergentes et les oppositions entre les principaux acteurs, voir particulièrement : Aguiton C., et al., Où va le monvement altermondialisation ?... et autres questions pour comprendre son histoire, ses débats, ses stratégies, ses divergences, Paris, La Découverte, 2003, ainsi que d'autres témoignages pouvant être consultés, parmi lesquels Aguiton C., Le Monde nous appartient. Porto Alegre, Florence, Evian: les acteurs d'une autre mondialisation, Paris, Plon, 2001 ; Leite J.C., Gil C., Fórum Social Mundial : A bistória de uma invenção política [Forum social mondial : l'histoire d'une invention politique], São Paulo, Editora Fundação Perseu Abramo, 2003. 
(Association brésilienne d'organisations non gouvernementales), le FBOM (Forum des ONG et mouvements sociaux pour l'environnement et le développement) et l'IBASE (Institut brésilien d'analyses sociales et économiques).

Au sein de ce deuxième pôle, le degré d'hétérogénéité est plus grand parce que la diversité des ONG est, elle aussi, plus élevée que dans d'autres types d'organisations, comme les syndicats. Ainsi, les « thèmes » ou les « secteurs » d'action ne peuvent être de bons indicateurs. Dans leur majeure partie, les ONG s'inscrivent dans le pôle dit « de gauche ». Leur action est soit tournée vers des enjeux écologiques, soit vers l'assistance sociale, ou encore, elle peut être consacrée à l'expertise, au sens large. La constitution même de l'ABONG vient de l'effort d'un ensemble d'ONG de distinguer leur position par rapport à d'autres. Par ailleurs, la plupart de ces ONG maintiennent un lien avec l'Eglise catholique. Par exemple, pratiquement toutes les ONG fondatrices de l'ABONG ont une connotation religieuse (CEDI, FASE, IBASE, IECLB/CAPA, notamment ${ }^{20}$ ). Mais cela ne rend pas les intérêts et les bases de légitimation de ces ONG moins complexes. Comme cela a pu être souligné par Hélène Rivière O. d'Arc :

« les vrais héritiers des chemins tracés par l'Eglise des pauvres, par les associations et les regroupements communautaires territorialisés, etc., ont été les ONG, qui s'appuient de façon décisive, ou essentiellement, sur les anciens réseaux constitués par les $\mathrm{CEB}$, transmettant leurs propositions humanitaires ${ }^{21}$.»

D'autre part, en tant que groupes d'intérêt, elles maintiennent des relations différenciées avec les ONG internationales, avec leurs subordonnés au contexte local et avec les autres sphères d'action.

Un troisième pôle est constitué par des organisations de «mouvements sociaux », où se détachent le MST (Mouvement des travailleurs agricoles sans terre), la CMP (Centrale de mouvements populaires), et la CONAM (Confédération nationale des associations d'habitants). Là encore, à l'exception du MST (une des organisations les plus influencées par des proches de l'Eglise catholique), beaucoup de ces organisations sont en réalité des groupements de plusieurs organisations. En général, ces regroupements se font au nom d'une population «pauvre » ou exclue du marché officiel du travail.

Un quatrième pôle regroupe des organisations liées aux mouvements de femmes, comme l'AMB (Association de femmes brésiliennes) et la Marche mondiale de femmes. Le cinquième pôle est formé par l'association défendant les

20. Voir http://www.abong.org.br/, page consultée le 13 avril 2007.

21. D’Arc H.R.O., "Basismo” acabou? Análise sobre a participação comunitária no Brasil (19761990) [Le "Basisme », c’est fini ? Analyse sur la participation communautaire au Brésil], Cadernos CRM, Salvador, n²6-27, janvier-décembre 1997. 
droits des entrepreneurs, CIVES (Association brésilienne d'entrepreneurs pour la citoyenneté). Le sixième pôle rassemble les organisations rattachées au militantisme étudiant, où se détachent l'UNE (Union nationale d'étudiants) et l'UJS (Union de la jeunesse socialiste). Comme nous le verrons en détails par la suite, ce type d'organisations liées à la « jeunesse » réunit une partie quantitativement très importante du public du FSM, particulièrement celle d'origine géographique moins éloignée. Le septième pôle est composé d'une série d'organisations tournées sur des activités légèrement distinctes, mais toutes directement rattachées à l'Eglise catholique, soit en termes institutionnels, soit idéologiques. C'est le cas de Caritas Brésil, de la CBJP (Commission brésilienne de justice et paix de la CNBB), de l'IPF (Institut Paulo Freire), du Jubilé Sud Brésil et du Réseau Brésil de droits humains. Cette représentativité décisive d'organisations d'origines religieuses a même entraîné la création de l'un des 11 «espaces » du 5e FSM, consacré à « l'éthique, aux cosmovisions, et à la spiritualité 22 ». Au FSM de 2007, à Nairobi, elles ont mis en place le Forum mondial de la théologie de la libération ${ }^{23}$. Sans prétendre être exhaustif dans la liste des organisations, nous pouvons dire que celles-ci jouent un rôle non négligeable dans la promotion du FSM, sans pour autant être véritablement intégrées localement, en raison de leur création récente ou du fait de leur association plus directe aux organisations de niveau international. C'est notamment le cas d'ATTAC, dont les représentants, et ce n'est pas une coïncidence, se trouvent parmi les plus proches de l'«altermondialisme » au sens d'une opposition à la mondialisation néolibérale.

Cette pluralité d'organisations et de sujets, ou de «causes", forme un «champ multi-organisationnel » $\mathrm{d}$ ' «alliance et de conflit » 24 avec des positions bien spécifiques à l'égard de la configuration de l'espace politique. En général, il s'agit du pôle de « gauche » dans les différentes sphères sociales et organisations rassemblées au FSM, comme c'est le cas du syndicalisme, du champ religieux, des ONG, etc. (à l'exception de la politique "partisane », étant donné que participer au FSM au nom d'un parti politique est interdit). Cela ne saurait toutefois rendre compte du problème en question, notamment parce que, outre des organisations, différents types de ressources, principes de légitimation, modalités d'engagement et de militantisme entrent en confrontation. Cela n'est pas sans conséquences pour les projets d'interprétation du « social » dans leurs relations avec la "politique », allant au-delà de l'opposition généralisée à la « mondialisation ». A première vue, on pourrait penser qu'il s'agit d'une opposition de principe, comme c'est le cas de la représentation syndicale à l'égard de positions et d'activités philanthropiques et d'assistance. Il s'avère que, bien que leurs définitions tournent autour de l'idée de défense des intérêts de "classe », le syndicalisme n'est pas réductible à la représentation abstraite « des classes » et que

22. Voir Hernandez A., «Etica, cosmovisões e espiritualidade » [Ethique, cosmovisions et spiritualité], in Blin A., et al., 100 Propostas do Fórum Social Mundial, Petrópolis, Vozes, 2006.

23. Voir http://www2.wftl.org. Page consultée le 12 janvier 2007.

24 . Agrikoliansky E., Fillieule O., Mayer N., op. cit., p. 18. 
les mouvements dits « sociaux ", tout comme les ONG, agissent comme des groupes d'intérêt. En se constituant à des degrés variés comme organisations d'assistance et comme groupes d'intérêt, les modalités de « représentation» de ces intérêts sont diverses, généralement avec la prédominance de l'expertise et ou même du lobbying ${ }^{25}$. Malgré des tensions évidentes à l'intérieur de cet ensemble de ressources et de légitimation, il y a donc plus de complémentarité et d'ambivalence que d'exclusivité. Certes, le format du FSM comme « espace » œuvre en ce sens, mais il faut prendre en compte les intérêts concrets et les logiques dans lesquelles s'inscrivent ces organisations. En ce qui concerne les ONG, il s'agit de celles situées dans des configurations sociales et politiques spécifiques qui, pour la plupart, œuvrent dans un contexte national ou local dans les pays du Tiers-monde, et donc dans une situation autre de celle des grandes ONG internationales ${ }^{26}$. L'assistance et les valeurs associées comme principes de légitimation, ainsi que les ambivalences relatives à l'espace politique, caractérisent d'ailleurs les grandes ONG des pays développés 27 . Cependant, dans le domaine ici étudié, il est nécessaire de prendre d'abord en compte le renforcement de la philanthropie et de l'expertise dans leurs versions nord-américaines, particulièrement depuis la fin de la Guerre froide, ainsi que les nouvelles conditions de relations entre centre et périphérie. Ensuite, ces nouveaux « universaux » qui sous-tendent la philanthropie comprennent l'ensemble des valeurs, avec le reforcement d'une conception « gestionnaire » des conflits sociaux et politiques et des relations de dépendance (financière, institutionnelle, culturelle) des ONG locales envers les ONG internationales et les organisations multilatérales 28 . Cette opposition entre des principes de légitimation basés sur la philanthropie ou l'assistance et une conception « gestionnaire » des conflits sociaux et politiques est flagrante, même dans les débats promus par l'ABONG ${ }^{29}$. Néanmoins, ce type d'oppositions, même s'il est explicite, est limité plus directement aux discussions intellectualisées ou aux leaders de chaque pôle, ce qui, évidemment, n'exclut pas les controverses entre représentants de ces différentes organisations. D'autre part, toutes les catégories en confrontation (comme celles associés à l'assistance, à l'intermédiation d'intérêts, à l'expertise, etc.) peuvent être présentées et saisies dans un sens absolument positif ou négatif, selon les positions ou les contextes respectifs dans lesquels elles s'inscrivent.

25. Sur les activités d'expertise dans la représentation d'intérêts, voir Offerlé M., Sociologie des groupes d'intérêt, $2^{\mathrm{e}}$ éd., Paris, Montchrestien, 1998, pp. 118-122, et sur les utilisations de l'expertise et du scandale par les ONG humanitaires, voir Siméant J., Dauvin P. (dirs), op. cit., pp. 24-29.

26. Pour une étude des ONG internationales, voir particulièrement Cohen S., «ONG, altermondialistes et societé civile internationale ", Revue française de science politique, Paris, vol.54, $\mathrm{n}^{\circ} 3$, juin 2004.

27. Siméant J., Dauvin P. (dirs.), op. cit.

28. Pour plus de détails, voir Dezalay Y., Garth B.N., op. cit.

29. Sur ces projets d'interprétation et la polysémie qui en découle, voir particulièrement Arantes P.E., " Esquerda e direita no espelho das ONG » ["Gauche et droite au miroir des ONG »], Cadernos Abong, São Paulo, n²7, mai 2000. Pour des considérations sur l'hétéro- 
Néanmoins, de telles oppositions dépendent plus du type d'organisations en présence et des positions de chacune vis-à-vis de l'espace politique que de la confrontation de principes abstraits. En deuxième lieu, comme on verra, les ONG sont dépendantes des ressources financières octroyées par des grandes ONG et, d'autre part, par des organismes gouvernementaux nationaux. Cela forme un continuum dont une extrêmité est constituée par la philanthropie d'entreprise, quasiment exclue du FSM, et par d'autres modalités de philanthropie et d'assistance sociale, comme celles des églises et des ONG internationales. L'extrémité opposé est formée par les ONG à un caractère plus militant, dont les activités se concentrent sur l'expertise et les mouvements sociaux. Afin de mieux comprendre le FSM, outre ces divisions et conditions relatives aux modalités d'engagement et à leurs fondements idéologiques respectifs, il convient de prendre en considération les différents niveaux de participation. Synthétiquement, une série de référentiels généraux se présentent comme de grands «sujets", parmi lesquels se trouvent ceux associés au tiersmondisme. Les pratiques ou les «activités » associées à ce niveau plus général tendent à se concentrer sur les grandes « conférences ». Par ailleurs, la participation est tout de même une opportunité - individuelle ou collective - d'insertion dans des réseaux de différents niveaux où s'articulent alliances et oppositions autour d'un axe, sujet ou secteur d'action particulier. Cette dynamique tend à instaurer des « réseaux verticaux en cascade », dans lesquels un pôle inclut des organisations internationales, jusqu'à atteindre celles dont l'action est davantage « locale». De ce fait, l'appropriation et l'usage des différents référentiels idéologiques prennent sens selon l'insertion dans des réseaux de niveaux national et international.

Ainsi, et bien que cela puisse paraitre surprenant, il est possible de comprendre que "l'altermondialisme ", pris comme définition du $2^{\text {e }}$ FSE ${ }^{30}$, demeure un terme qui est loin de faire sens pour la quasi-totalité des personnes interrogées. Les rares exceptions sont les dirigeants les plus actifs des grandes organisations, en général promotrices du FSM, ayant un fort parcours militant et des expériences internationales. Les significations des critiques portées contre la «mondialisation » sont d'origines très diverses, comme celles restreintes aux effets d' "exclusion sociale ", dont la revendication principale est la réduction des inégalités pour les « consommateurs » ou un « respect de la diversité ». Ceci est généralement exprimé par des représentants d'organisations directement liées à la philanthropie et à l'assistance, passant par les différentes positions de militantisme d'expertise. D'un autre côté, la critique du capitalisme s'exprime au nom d'un principe de «solidarité », généralement fondé sur la morale catholique, jusqu’aux positions anticapitalistes plus direc-

généité de la "nébuleuse » altermondialiste et les conditions propres aux pays du « Nord » face à ceux du «Sud » au FSE, voir Gobille B., Uysal A., op. cit., pp. 124-125.

30. Voir Agrikoliansky E., Sommier I. (dirs.), op. cit. 
tement liées aux organisations de « gauche », proches des mouvements sociaux et du syndicalisme.

Lorsqu'on essaye de comprendre les différences relatives qui s'expriment dans les tentatives de définition de la «mondialisation ", on distingue plusieurs éléments, et en premier lieu la position de l'organisation dans le spectre des luttes idéologiques et dans ses relations avec l'espace politique. Ce premier élément d'explication est lié au type d'organisation, aux parcours respectifs, aux modalités d'engagement de leurs membres, et surtout aux principes de regroupement et de légitimation des organisations. D’un côté, à " gauche », se situent quelques mouvements sociaux et syndicats. Dans le pôle opposé, celui des ONG, les organisations se consacrant à l'assistance se limitent à offrir « quelques remèdes à l'intérieur de la société, pour renforcer le système qui exclut ", même le «système néo-libéral » ${ }^{31}$, selon les termes d'un représentant de "gauche »(MST). De manière presque similaire, un autre représentant du pôle de "gauche » (CMP) oppose à la «mondialisation » économique, celle de la « classe ouvrière » et des «mouvements populaires » et, dans une allusion aux organisations philanthropiques, il souligne qu'il s'agit d'une «lutte » par «miettes » et que «cette lutte ne sert qu'au capitalisme international ${ }^{32}$. Ces différentes positions sont reconnues, même par des représentants d'organisations opposées comme le MNDH, dont le représentant affirme : «les ONG ont une position et les mouvements sociaux, une autre ». Néanmoins, selon cette perspective, "les ONG ont une capacité de lecture plus recherchée, plus technique", "elles sont plus développées que les mouvements [qui] font une évaluation plus politique [davantage] basée sur des idées que sur des données " 33. Se pose donc ici la question d'un projet d'évaluation qui mettrait positivement l'accent sur la «technique » (ou l'expertise) en opposition à la « politique », ou la capacité de mobilisation comme ressource de légitimation.

Une autre manière de justifier la position des ONG opposées à la politique consiste à appréhender l'ensemble des ONG comme soumises à une division du travail, avec différentes modalités d'engagement. Ainsi, ce qu'on appelle le «troisième secteur » est divisé en trois grands blocs, dont le premier est constitué par des ONG de défense de droits, généralement affiliées à l'ABONG, le deuxième par celles à caractère philanthropique ou d'assistance, comme les organisations proches de l'Eglise et le troisième, par les fondations qui agissent sur le plan de la responsabilité sociale des entreprises ${ }^{34}$. Dans cette perspective, la défense de «droits » est bien évidemment la modalité la plus facilement légitimée au sein du FSM. Pour un grand nombre de ces organisations d'aide, d'assistance et de responsabilité sociale des entreprises,

31. Entretien avec J., 29 janvier 2005 à Porto Alegre.

32 . Entretien avec L., 27 janvier 2005 à Porto Alegre.

33 . Entretien avec R., 28 janvier 2005 à Porto Alegre.

34 . Entretien avec F., 28 janvier 2005 à Porto Alegre. 
comme nous l'avons vu, les problèmes relatifs à la mondialisation peuvent être réduits au "respect de la diversité ». Selon les termes d'un représentant d'Interage (un conglomérat de grandes ONG internationales), «il y a un aspect positif [dans la] mondialisation ", mais "pas comme elle se présente » actuellement, parce qu'il est nécessaire de "respecter la diversité [ou de] préserver les cultures des régions", étant donné que "les gens ne seront pas égaux» ${ }^{35}$. De même, un représentant de Greenpeace souligne : "[c']est un bon exemple que la mondialisation peut fonctionner, parce qu'on respecte les intérêts de chaque pays [...], on fait des partenariats 36 ».

Cependant, outre la position de chacun dans l'espace politique, les schémas d'évaluation relatifs à la « mondialisation »sont directement associés à la position dans la hiérarchie des organisations où, d'un côté se trouvent les représentants de petites ONG, de «mouvements sociaux » ou de syndicats centrés sur l'action « locale » et où, de l'autre côté, figurent les représentants de grandes organisations nationales et surtout internationales, pour lesquelles la « mondialisation » est une question importante. Cela permet même d'exercer une position de supériorité et un pouvoir de censure à l'égard du caractère local des premières, sans pour autant éliminer les ambivalences qui dépendent de la position respective de chacun dans l'espace des luttes politiques et idéologiques. Pour ne citer qu'un exemple, une de ces grandes organisations internationales, l'Action Aid, dont la représentante fait "partie de cette mobilisation pour une autre mondialisation, [...] aux côtés d'autres organisations internationales, [...] étant à l'avant-garde de cette conception qu'est la transformation mondiale ». Ceci la rendrait :

« différente d'autres organisations qui ont leur vision locale très précise, très délimitée, mais qui ne réussissent pas, ne s’inquiètent pas, ne parviennent pas toujours à projeter une transformation sur un plan plus important. [...] La majorité des ONG se tourne sur le local, tout au plus sur le national 37 ».

Bien que cela ne puisse pas être ici détaillé, il semble évident que les différentes perspectives à l'égard de la « mondialisation » relèvent de la projection de divers schémas d'interprétation $\mathrm{du}$ «social » et de croyances politiques. Mais il est aussi question des différentes modalités d'engagement, qui renvoient à d'autres univers sociaux, notamment ceux des luttes syndicales, de représentation d'entreprise, du champ religieux, etc.

Les relations avec l'espace politique sont plus faciles à saisir, en dépit de variations. Sur ce point également, une première condition tient aux positions 
respectives des organisations dans le spectre des luttes politiques et idéologiques et face à l'espace politique, ce qui est directement associé au parcours social, aux "affinités " politiques et aux intérêts dans l'engagement ou à la prise de distance des activités politiques de leurs membres ${ }^{38}$. Entrent en jeu également les modalités de "politisation 39 », les intérêts et objectifs qui peuvent être plus politiques, au sens strict, ou bien davantage centrés sur la morale civique qui défend des valeurs comme par exemple le « renforcement de la société civile». Enfin, la notion de politique est aussi associée aux partis, ce que la rend moins légitime. On observe même un effort généralisé pour exempter les organisations de soupçons de liaisons partisanes et ainsi de la possibilité d'une «instrumentalisation» des organisations par les partis. De plus, bien que beaucoup soit évidemment affiliés à un parti (en général au PT, qui a le plus d'affinités avec les militants rassemblés au FSM), cela est de plus en plus accepté, si et seulement si cette affiliation est présentée comme relevant de la sphère privée. De surcroît, la politique partisane est fréquemment présentée comme liée aux intérêts « personnels » des leaders. Le représentant d'ATTAC au Brésil, par hasard une organisation politique à caractère plus général, est la seule personne interviewée qui considère les partis politiques comme des alliés. Comme l'explique ce représentant, qui a un long parcours militant étudiant et politique, «toutes les entités, organisations sociales et partis politiques qui soutiennent les propositions que nous défendons sont nos partenaires. Nous n'établissons aucun type de restriction aux partis politiques 40 ».

Mais, dans ce cas, il est nécessaire d'intégrer ces perspectives dans certaines logiques d'action et certains intérêts. Johanna Siméant ${ }^{41}$ suggère que, dans le cas des ONG humanitaires, outre la socialisation primaire et secondaire, le caractère apolitique des militants provient d'une logique de démarcation face aux modalités d'engagement concurrentes, de la nécessité d'une certaine «neutralité politique » comme condition préalable à l'intervention dans des contextes de conflits et aux bonnes relations avec les agents gouvernementaux, comme condition du succès des campagnes de collecte de fonds et comme possibilité de cohabitation dans un univers composé de différentes orientations politiques. Dans le cas en question, il ne s'agit en général pas à proprement parler d' « apolitisme », mais bien de modalités spécifiques de définition et d'action «politique », basées notamment sur deux conditions. On a d'abord la dépendance généralisée à l'égard des grandes ONG et autres organisations internationales qui représentent des sources de financement non négligeables et, ainsi, un évident «marché » de « projets ». Ensuite, et manière plus généra-

38. Siméant J., « Un humanitaire “apolitique” ? Démarcations, socialisations au politique et espaces de la réalisation de soi », in Lagroye J. (dir.), La Politisation, Paris, Belin, 2003, p. 195.

39. Lagroye J., "Les processus de politisation ", La Politisation, op. cit.

40. Entretien avec J., 30 janvier 2005 à Porto Alegre.

41. Voir Siméant J., Dauvin P. (dirs.), op. cit.; Siméant J., «Un humanitaire "apolitique" ? Démarcations, socialisations au politique et espaces de la réalisation de soi », op. cit. 
lisée, il s'agit d'une dépendance envers les organismes publics, principales sources de financements des ONG au Brésil.

Sur ce point, on peut également citer, en tant que figure du pôle plus politique, un représentant du MST, selon lequel les ONG qui «travaillent avec des pawvres », comme Caritas, seraient tournées vers les «premières aides », à travers de «petits projets» ou des «dons». A cela, s'oppose une position de mobilisation, consistant à « convoquer le citoyen pour une lutte plus grande 42 ». De même, un représentant de la CMP souligne que «la relation avec la politique, [...] c'est tout ce que nous avons fait». Quant aux ONG, elles « ont un problème, parce qu'il existe les mouvements populaires, les mouvements sociaux, les ONG et il $y$ a des ONG qui n'ont aucun engagement avec les transformations de la société [et qui] se montrent dehors [à l'extérieur] comme si elles détenaient la vérité » ${ }^{43}$. A l'autre bout du spectre se trouvent des organisations comme Caritas, pour laquelle le "profil de [son propre] personnel [...] doit être militant », étant donné qu'il s'agit «de défendre un projet de vie ». Sans pour autant être politiquement orienté, ce profil doit être orienté par une notion de militance, afin de se «battre pour un monde meilleur et [...] avoir la mystique et la spiritualité de l'Eglise ${ }^{44}$.

Mais, comme mentionné plus haut, la politique la plus forte et généralement exclue des relations légitimes, c'est l'action "partisane », généralement associée à un certain cloisonnement ou à des «intérêts personnels». Par exemple, pour la représentante d'un conglomérat d'ONG internationales (Action Aid 45), ayant milité au sein d'organisations catholiques, les partis politiques constituent «une demi-douzaine de leaders qui finissent par s'emparer des principes et des idées", contrairement aux ONG qui réussiraient «à créer leur propre espace et leur propre champ d'action [et qui] ont un projet concernant l'Etat », étant donné qu'elles représentent «un bras important de la société pour construire des changements dans le champ dans lequel elles agissent ». Pour cela, l'objectif central est $\mathrm{d}$ '« influencer » les politiques gouvernementales ${ }^{46}$. D'autre part, la politique est considérée comme «influente » sur des organismes et agents gouvernementaux, et comme «formatrice de citoyens». Ainsi, Greenpeace, comme l'explique l'un de ses représentants, mène "une action très forte dans l'environnement politique », bien qu'il ne reçoive pas l'aide de partis politiques ou de gouvernements. "[Greenpeace] cherch[e] toujours à bien connaître les politiques publiques», en vue de la réalisation de campagnes de pression pour la modification ou l'application de la législation ${ }^{47}$. De la même façon, la représentante d'Interage souligne :

42. Entretien avec J., 29 janvier 2005 à Porto Alegre.

43 . Entretien avec L., 27 janvier 2005 à Porto Alegre.

44 . Entretien avec R., 28 janvier 2005 à Porto Alegre.

45. Voir http://www.actionaid.org.br/

46. Entretien avec R., 30 janvier 2005 à Porto Alegre.

47. Entretien avec G., 28 janvier 2005 à Porto Alegre. 
« Nous sommes des êtres politiques, on ne peut pas nier la politique. Le Forum social mondial, c'est cela. On n'agit pas sur la question partisane mais sur la pensée politique, avec la certitude d'influencer $[\ldots]$ les politiques publiques 48 ».

La définition de la politique unanimement défendue et au nom de laquelle sont justifiées les relations avec cet univers est donc synonyme d'action publique et de politiques gouvernamentales, qui acquièrent une signification propre. Cela résulte, en premier lieu, de la dépendance à l'égard des ressources octroyées par les gouvernements et partis politiques, mais c'est aussi la conséquence des activités d'intermédiation d'intérêts réalisées par ces organisations. Etant donné qu'elles agissent pour obtenir des ressources gouvernamentales, les relations directes avec le gouvernement peuvent avoir plus d'efficacité que celles qui se font par l'intermédiaire des partis. Finalement, une autre notion de politique défendue par les représentants des ONG associe celle-ci à l'intermédiation d'intérêts au sens de la défense de droits, à travers l'expertise notamment. Cette notion de politique est généralement présentée au nom de certains principes de morale civique, comme la défense de la "citoyenneté » (une notion devenue des plus polysémiques), du renforcement de la «société civile 49 »et sur lesquels peuvent reposer des « projets » divers, mais plus particulièrement ceux menés dans le domaine de l'«éducation ».

\section{Les participants brésiliens}

La distance géographique et les rapports avec les réseaux déjà intégrés dans le FSM ont pour effet de différencier les participants selon leur lieu d'origine. En prenant en compte exclusivement les participants brésiliens (qui représentent $29,06 \%$ de notre échantillon de 2540 personnes sélectionnées de façon aléatoire), nous pouvons mentionner certaines de leurs principales spécificités par rapport aux autres participants.

Les participants brésiliens entretiennent un rapport privilégié avec des organisations locales, moins avec les organisations nationales et internationales, contrairement notamment aux Européens. En moyenne, les participants brésiliens sont bien plus jeunes et parmi les professions représentées on trouve une majorité de fonctionnaires et d'étudiants et une faible proportion d'employés d'ONG/d'organisations de la société civile/parti politique ${ }^{50}$. On retrouve également plus d'individus n'ayant qu'un diplôme de niveau secondaire. Il y a enfin une plus grande proportion de syndicats et $d^{\prime}$ " associations de classe 51 »

48. Entretien avec T., 27 janvier 2005 à Porto Alegre.

49. A propos de l'emploi de notions telles que "société civile » et de leurs implications normatives, voir Siméant J., Dauvin P. (dirs)., op. cit., pp. 10-19.

50. Ces categories ont été mises ensemble dans la formulation du questionnaire.

51. Selon la législation brésilienne, outre les syndicats, il existe aussi des «associations de 
(19,7\%), et les ONG sont beaucoup moins représentées parmi ces participants. D'autres indicateurs pertinents peuvent venir corroborer ces differences.

En effet, si l'on prend les principales variables (sexe, âge, degré de scolarisation, profession actuelle, participation à un mouvement ou à une organisation sociale, nature institutionnelle de ce mouvement ou organisation et filiation à un parti politique), à l'exception de la division par genre qui est presque équivalente, on trouve une forte relation entre leurs catégories et quelques tendances très claires semblent se dégager ${ }^{52}$. Il est ainsi possible de caractériser les principales catégories de participants ainsi que les principes de leurs oppositions. Le premier axe d'opposition observé tient essentiellement à la participation ou non à un mouvement ou une organisation sociale, à la profession, à l'âge, au degré de scolarisation et à l'affiliation à un parti politique. Deux catégories peuvent être distinguées : la première, constituée essentiellement d'étudiants âgés de 18 à 24 ans, qui ne participent à aucun mouvement ou organisation sociale, non affiliés à un parti politique, suivant une formation supérieure, majoritairement féminine. A un moindre degré, cette catégorie est complétée par des individus âgés de 14 à 17 ans, cumulant de 9 à 12 années de scolarité.

A ce pôle plutôt « étudiant » s'oppose un autre pôle, caractérisé par un lien important avec la politique. Ses membres participent aux organisations et aux mouvements sociaux; ils sont affiliés à un parti politique et sont, dans une large mesure, également sympatisants d'un syndicat, d'une « association de classe »,ou d'une ONG. La profession la plus représentée est celle des fonctionnaires, puis celle des employés d'ONG/organisation de la société civile/parti politique. Les moyennes d'âge sont plus élevées que celles du pôle opposé, avec des individus âgés de 35 à 44 ans et de 45 à 54 ans. On observe enfin un plus fort taux d'achèvement des études supérieures et une prédominance du sexe masculin.

Une série de plus de 40 variables à caractère idéographique montre des degrés d'association très bas par rapport aux variables liées aux origines sociales, à la profession et au diplôme scolaire. Ceci est sûrement dû au fait que les participants du FSM constituent une population préalablement sélectionnée, avec un fort degré d'homogénéité idéologique, du moins d'une manière générale, si l'on n'entre pas trop dans les détails. Les questions présentées se limitent justement à ce niveau général, elles ne prennent ni en compte les clivages subtils mais importants, ni les oppositions observables au sein de cette popu-

classe » qui représentent des catégories professionnelles spécifiques.

52 . En guise de première exploration, un test d'analyse des correspondances multiples a été appliqué en considérant ces variables comme actives et celles de caractère idéographique comme illustratives. Ce test démontre un fort degré de corrélation entre les variables actives, avec $12,57 \%$ de valeur propre dans le premier axe factoriel, 6,87 \% dans le deuxième, 6,43 \% dans le troisième et $6,22 \%$ dans le quatrième axe. 
lation autour de questions plus concrètes. Ceux qui se placent dans ce pôle se désignent majoritairement eux-mêmes comme étant de "gauche ». Ils valorisent aussi l'exécution de politiques par l'Etat, au détriment des « organisations de la société civile » et du « contrôle » des politiques par ces organisations.

La troisième catégorie se constitue essentiellement d'étudiants liés à l'espace politique. Ses membres se caractérisent par leur formation supérieure inachevée et par leur rattachement à un parti politique ou à une ONG. Agés de 18 à 34 ans, ils sont étudiants ou employés d'ONG/organisation de la société civile/parti politique, et majoritairement de sexe masculin. Le pôle opposé est formé par un ensemble d'individus âgés de 14 à 17 ans et par un autre ensemble âgé de 45 à 64 ans, ces deux groupes ayant un niveau de scolarité relativement bas. La plupart sont sans emploi ou ont des professions indéfinies (catégorie « autre » dans notre questionnaire). D'autres, en plus faible proportion, sont fonctionnaires, membres de mouvements sociaux populaires et, dans une moindre mesure, de syndicats ou d'« associations de classe ». En termes idéologiques, le pôle « étudiant » politique se caractérise par son jugement plus négatif envers les institutions comme l'Organisation mondiale du commerce (OMC), le Fonds monétaire international (FMI) et la Banque mondiale. Les membres du pôle opposé abordent, eux, la mondialisation d'une manière plus positive (possibilité de connexions entre la société civile au niveau mondial, davantage d'opportunités à saisir pour tout un chacun, etc.).

La cinquième catégorie est constituée par ceux qui sont affiliés à un parti politique. Ses membres sont majoritairement des hommes, participant à des syndicats ou à des « associations de classe ». Dans une moindre mesure, ils sont étudiants, avec un niveau scolaire relativement bas. Ce pôle est donc essentiellement constitué d'une sous-catégorie plus fortement associée aux fonctionnaires, syndicalistes, affiliés à un parti politique, âgés de 45 à 54 ans, et par une seconde sous-catégorie, plus "étudiante », qui ne participe pas à des organisations ou mouvements sociaux, âgés de 18 et 24 ans. Ces deux sous-catégories s'opposent au pôle formant une sixième catégorie, dans lequel se démarquent ceux qui sont membres actifs d'une ONG et qui sont employés d'ONG/organisation de la société civile/parti politique, avec une prédominance du sexe féminin. On y treouve une majorité d'individus âgés de 25 à 34 ans ou de 55 à 64 ans, ayant un niveau d'études élevé (diplôme d'études approfondies, doctorat ou études supérieures achevées). C'est dans ce pôle que l'on trouve la plus grande représentation de professions indépendantes ou libérales ou de professions mal définies («autres»). En termes idéologiques, les individus de ce pôle sont les plus affiliés aux partis politiques et sont plus favorables aux partis eux-mêmes, au pouvoir exécutif, aux syndicats, aux « associations de classe », ainsi qu'au système électoral. Ils se désignent dans une très large mesure comme de " gauche » et ont une vision plus négative de la justice, de l'ONU et même des ONG.

Il s'agit donc d'une population qui se caractérise principalement par la forte 
capitalisation de certaines ressources, parmi lesquelles figurent un haut niveau d'études et l'appartenance à des catégories professionnelles spécifiques. En matière de profession, outre la forte présence d'étudiants ${ }^{53}$, on retrouve beaucoup de fonctionnaires $(21,9 \%)$, pour ce qui est des origines sociales et plus spécifiquement de la profession du père $(24,5 \%)$ et de la mère $(20,3 \%)$. Mais, comme cet univers est un « espace » fédéré, avec une forte participation de militants et d'institutions centrées sur des activités d'expertise et d'advocacy, ou d'intermédiation d'intérêts en général, sur la philanthropie, l'aide et l'assistance, d'autres sur la représentation syndicale et d'autres encore sur des mouvements sociaux tournés sur les segments les plus divers,on y trouve là aussi une très forte hétérogénéité.

L'engagement et le militantisme - dans des organisations, des mouvements sociaux, dans des ONG ou des partis politiques - est un des principaux éléments qu'ont en commun les participants du FSM. Ce militantisme possède des liens étroits avec les indicateurs d'insertion et de position sociale. Ainsi, la participation dans les organisations ou mouvements sociaux permet de maintenir une forte liaison avec, en premier lieu, la profession exercée. D’un côté, se placent les employés d'ONG/organisations de la société civile/parti politique se démarquent $(17,3 \%$ contre $0,6 \%$ du total), suivis des fonctionnaires $(27,7 \%)$, et d'autres, dans une moindre proportion, comme les retraités. D'un autre côté, avec une plus faible participation, apparaissent les étudiants et les chômeurs. Cette participation aux organisations ou mouvements sociaux va souvent de pair avec le fait qu'un membre de la famille de l'individu soit, lui aussi, militant ${ }^{54}$. Le même phénomène se produit avec l'affiliation à un parti politique. En effet, parmi les participants à un mouvement ou à une organisation sociale, on trouve une proportion plus élevée de personnes affiliées à parti politique (39,5\% contre $27,3 \%$ du total). Cela montre bien que, même s'ils ne sont pas réductibles à cela, et s'ils peuvent parfois même y être opposés, le militant associatif et le partisan sont interdépendants et, parfois, complémentaires.

La filiation partisane, en tant que modalité d'engagement, configure un type spécifique de participants du FSM et manifeste une relation avec la division des participants par genre. Comme nous l'avons déjà mentionné, il existe une majorité très nette d'hommes parmi les affiliés à un parti $(61,9 \%$ contre $48,5 \%$ du total).

Concernant la profession, on observe également des adéquations marquées avec la filiation partisane. Cette adéquation est surtout visible chez les

53. Dans le questionnaire distribué, le statut d'étudiant a été présenté comme une réponse de profession possible

54. Selon d'Arc, plus « qu'un clientélisme dans sa signification la plus stricte ", les ONG « contribuent à l'apparition de lobbies, susceptibles de passer par des alliances ad hoc" (voir d'Arc H.R.O., op. cit.). Cela n'exclut pas la cooptation à travers différents réseaux, dont ceux basés sur les liens de parenté. 
fonctionnaires (34,2\% contre 21,6\%), les employés d'ONG/organisation de la société civile/parti politique $(15,3 \%$ contre $10,7 \%)$, les retraités $(3,6 \%$ contre $1,8 \%)$ et les paysans $(2,6 \%$ contre $1,1 \%)$, ces deux dernières catégories n'étant que très faiblement représentées. Dans le pôle ayant moins d'individus affiliés, figurent notamment les étudiants (18,9\% contre $37 \%)$ et, malgré leur faible nombre, les chômeurs (2,6 \% contre 3,6 \%).

Les participants à un mouvement ou à une organisation sociale s'inscrivent également à des partis politiques dans une proportion bien plus grande $(81,6 \%$ contre $56,5 \%)$. La forme institutionnelle la plus étroitement liée à la filiation partisane est le syndicat et l'« association de classe ». Dans le pôle des individus proportionnellement les moins affiliés à un parti, se trouvent les membres d'ONG (20\% contre $33,4 \%$ ), et ceux qui participent à d'autres types de mouvements ou d'organisations sociales ( $5 \%$ contre $9,1 \%$ ). A l'instar d'autres modalités d'engagement, la filiation partisane ${ }^{55}$ dépend, elle aussi, fortement de la participation d'un autre membre de la famille à un mouvement ou à une organisation sociale $(61,5 \%$ contre $39,6 \%)$.

Finalement, la profession exercée, le degré de scolarisation et l'engagement sont autant de facteurs présidant à l'éventuelle participation au FSM, d'abord à sa préparation à travers la «consultation et agglutination» des probables participants. La participation à ce procesus de consultation est liée au degré de scolarisation, au niveau de la formation (taux d'individus ayant achevé leur formation supérieure : $37,2 \%$ contre $29,6 \%$ du total, ayant un diplôme d'études approfondies ou un doctorat: $15,9 \%$ contre $10,3 \%$ du total). Ceux qui ne participent pas à la préparation du FSM ont, majoritairement, une formation supérieure inachevée, c'est-à-dire qu'ils sont principalement encore étudiants. Cette participation au FSM, en amont ou en aval, dépend également de l'activité professionnelle actuelle. Parmi ceux qui ont contribué à la préparation du Forum, figurent au premier plan des employés d'ONG/organisation de la société civile/parti politique $(15,9 \%$ contre $10,5 \%$ au total), suivis des fonctionnaires $(26,9 \%$ contre $22,3 \%)$, des paysans $(2,8 \%$ contre $0,8 \%)$ et des retraités $(2,8 \%$ contre 1,8\%). Dans le pôle opposé, nous retrouvons les étudiants $(17,6 \%$ contre $37,2 \%)$, les employés de sociétés privées (7,6 \% contre 8,7 \%), les chômeurs $(2,8 \%$ contre $3,4 \%)$ et, enfin, ceux qui classés dans la catégorie « autres » professions $(2,8 \%$ contre $4,4 \%)$. Parmi ceux qui ont pris part à la préparation du FSM, il y a en effet une proportion beaucoup plus grande d'individus appartenant à un mouvement social $(72,7 \%$ contre $56,2 \%)$. De même, on trouve une plus grande proportion de personnes dont un membre de la famille participe à un mouvement ou à une organisation sociale ( $54,5 \%$ contre $39,7 \%$ au total). Finalement, parmi ceux qui ont aidé à la préparation du FSM, il y a une proportion bien plus grande d'individus qui se considèrent comme bien familiarisés

55 . Est considérée comme «filiation partisane » toute affiliation à un parti politique. 
avec la Charte de principes du forum (56,3\% contre $33 \%)$.

Concernant ce degré de familiarité avec la Charte de principes, une question spécifique a été posée aux participants. Comme la plupart des autres indicateurs d'engagement, celui-ci est directement associé aux niveaux de scolarisation 56 (bien que de manière non exclusive). Parmi ceux qui pensent être familiarisés avec cette Charte, se démarquent ceux qui possèdent un diplôme d'études approfondies ou un doctorat (15,3\% contre 10,4\%), une formation supérieure complète $(35,6 \%$ contre $29,1 \%)$ et, à un moindre degré, cinq à huit ans d'études $(5,5 \%$ contre 3,7 \%). Dans le pôle opposé, ressortent les individus ayant une formation supérieure non achevée $(28,4 \%$ contre $36,5 \%$ ) et neuf à douze ans d'études (14,4\% contre 19,7\%).

Ce degré de familiarité avec la Charte de Principes est, là aussi, fortement lié à la participation à un mouvement ou à une organisation sociale $(68,5 \%$ de ceux qui se considèrent familiarisés, $54,5 \%$ de ceux qui se considèrent «un peu familiarisés » et 47,2 \% de ceux qui ne se considèrent pas familiarisés du tout participent à un mouvement ou à une organisation sociale). Seuls 31,5\% de ceux qui se considèrent comme familiarisés avec la Charte de principes ne participent à aucun mouvement ou organisation sociale (contre $43 \%$ de l'ensemble total). Pour finir, ceux qui se considèrent familiarisés avec la Charte de principes attribuent une plus grande importance aux partis politiques.

Yves Dezalay et Bryant Garth avancent l'hypothèse selon laquelle l'intensification de l'importation de modèles culturels et institutionnels des EtatsUnis forme une nouvelle hiérarchie qui :

« comprend à son sommet l'élite des professionnels nord-américains [...] Cela se traduit dans le pays de la périphérie, par une hiérarchie professionnelle à deux niveaux. Le prestige d'une petite élite cosmopolite contribue à "provincialiser" une masse croissante de professionnels de second rang, qui ont profité de la multiplication des opportunités, durante la période d'après-guerre, pour acquérir des compétences spécialisés en droit, emn économie ou dans d'autres domaines 57 ».

L'un des principaux effets de ce phénomène sur le plan national serait la formation d'alliances pour la promotion d'expertises concurrentes, avec l'éventuelle opposition aux élites cosmopolites, accusées de «vendre l'Etat » sur le marché international. Par ailleurs, avec l'intensification des recours à la philanthropie comme moyen de légitimation, il y aurait une logique pyramidale de

56. La même chose a été constatée pour le 2e FSE. Voir Agricoliansky E., Sommier I., Hajji I.,

«Formes et publics de l'altermondialisme », in op. cit., p. 293.

57. Dezalay Y., Garth B.G., op. cit., pp. 110-111.

58 . Ibid., p. 207. 
diffusion/reproduction de l'expertise dans laquelle les intellectuels cosmopolites eux-mêmes s'attribueraient le rôle de porte-paroles des mouvements sociaux 58 . Comme semblent l'indiquer ces éléments, exposés ici brièvement, dans les nouvelles configurations où s'inscrivent les modalités d'engagement et de militantisme présentes au FSM, les hiérarchisations reposant sur l'expertise sont centrales. Néanmoins, il paraît également évident que ces nouvelles configurations dans les relations entre différentes modalités d'engagement, forces sociales et nouvelles questions légitimes, et, par ailleurs, dans les relations centre/périphérie, plus qu'elles ne le remplacent, résultent d'un processus d'ajout, avec une augmentation de l'hétérogénéité. L'approfondissement des efforts de compréhension de la multiplicité de logiques d'action requiert cependant que l'on inclue d'autres niveaux, outre les rassemblements du FSM déjà organisés. 